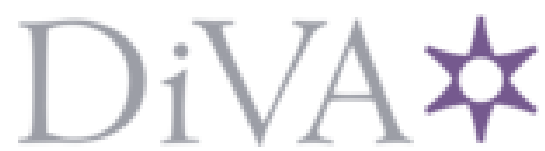

http://www.diva-portal.org

\title{
Postprint
}

This is the accepted version of a paper presented at ISGT Europe.

Citation for the original published paper:

Vardanyan, Y., Hesamzadeh, M R. (2016)

Modeling Regime Switching in Day-ahead MarketPrices Using Markov Model.

In:

N.B. When citing this work, cite the original published paper.

Permanent link to this version:

http://urn.kb.se/resolve?urn=urn:nbn:se:kth:diva-192884 


\section{Modeling Regime Switching in Day-ahead Market Prices Using Markov Model}

\author{
Yelena Vardanyan \\ Electricity Market Research Group \\ KTH Royal Institute of Technology \\ Email: yelena.vardanyan@ee.kth.se
}

\author{
Mohammad Reza Hesamzadeh \\ Electricity Market Research Group \\ KTH Royal Institute of Technology \\ Email: mrhesamzadeh@ee.kth.se
}

\begin{abstract}
The accurate price forecasting of electricity market is crucial for profit maximizing producers and consumers in liberalized power markets. In all market places (day-ahead, intra-day and real-time) accurate price prediction is needed to generate optimal bids and maximize the profit. This paper first presents three methods for forecasting day-ahead market prices, namely Generalized Autoregressive Conditional Heterosedastic (GARCH), Holt-Winter (HW) and Mean Reversion and Jump Diffusion (MRJD). These methods are based on three broad methodologies of time series analysis, exponential-smoothing and stochastic processes. The dynamics of hourly prices in day-ahead market are varying from day to day. Each forecasting tool is suitable to capture one type of price dynamics. To capture this phenomenon, we combine GARCH, HW and MRJD methods using proposed Markov switch. The proposed Markov model is tested using Nordic day-ahead prices.
\end{abstract}

Index Terms-Day-ahead prices, Markov switch, forecasting tools.

\section{INTRODUCTION}

The forecasting procedure of the electricity prices becomes more challenging with the liberalizing of electricity market. With the recent liberalization of power markets, electricity became a commodity that can be sold and bought at market price [1]. Moreover, electricity prices depend on daily demand cycles and unpredictable factors. One of recent unpredictable factors is renewable power, mainly wind and solar. In addition, the renewable power share is continuously increasing in the power system.

Power system stability requires continuous balance between production and consumption. One way to keep this balance is to store electric power and use it during peak demand periods. However, electric power is a special commodity that cannot be stored in an efficient way. For that purpose, electric batteries are needed, which are very expansive. Hence, to keep the balance between production and consumption in an effective manner the electric power should be generated in the very moment when the consumption takes place.

Impossibility to store electric power on one hand and increasing renewable energy share in the power system on the other hand cause high volatile market prices that is extremely difficult to forecast. Fig. 1 shows day-ahead market price volatility during one year (from March 102012 to March 10 2013) for Nordic power market [2].

Electricity is traded either in power exchange (in Nordic market it is called Nord pool) or with bilateral contracts. All

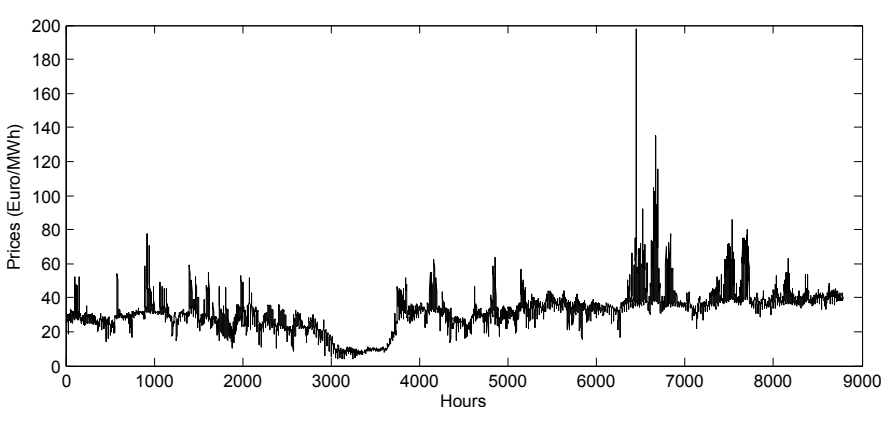

Fig. 1: Day-ahead market prices from March 102012 to March 102013.

day-ahead market participants are submitting hourly selling or buying bids to power pool before noon one day prior to the delivery day. A bid is a price-quantity pair which indicates how much electric power the market participants wants to sell or buy with the specified price. At noon day-ahead market is closed. Transmission system operator (TSO) arranges selling bids in ascending and buying bids in descending order; the cross point defines the hourly market prices. Profit maximizing power producers/consumers are willing to sell/buy electric power when the prices are high/low. Thus, having accurate electricity forecasting tools is of great interest for electricity market participants.

A big attention has been paid to develop forecasting models for day-ahead market prices. A detailed review on the topic is provided in the reference [3]. Time series analysis (or causal) models, exponential-smoothing models and stochastic models are three broad classes of techniques for forecasting day-ahead market prices.

The autoregressive integrated moving average (ARIMA) is a well-established model to forecast day-ahead market prices [4], [5]. Reference [6] predicts day-ahead market prices based on ARIMA methodology. The GARCH models are generalized ARIMA models, where the error term is considered time variant instead of having 0 mean and constant variance [7].

HW forecasting methods belong to the second class, which models the time series with unique or multiple seasonal patterns [8]. In reference [9] the authors use HW forecasting technique to predict electricity consumption. While the authors in [10] predict the expected imbalance cost in the real-time market using HW tool with a daily seasonal cycle. 
Mean Reversion \& jump diffusion (MRJD) is a forecasting tool which aims to capture not frequently happening spikes in day-ahead market price. Reference [11] predicts day-ahead market prices based on the MRJD methodology.

This paper presents three day-ahead market price forecasting alternative methods (GARCH, HW and MRJD) based on three broad methodologies respectively (time series analysis, exponential-smoothing and stochastic processes) and develops a Markov Switch to choose the best price predictor for the day of planning. The Markov switch is based on the Markov chain model. The flowchart of the whole framework is presented in Fig. 2. Day-ahead market historical price series from 10 March 2012 to 10 March 2013 is used to test forecasting models and is taken from Nord Pool [2].

The rest of paper is organized as follows. Section II discusses the GARCH predicting model for day-ahead market prices. Section III presents MRJD method. HW method is presented in section IV. Markov smart switch is introduced in section V. Section VI concludes the paper.

\section{Generalized Autoregressive Conditional HETEROSKEDASTIC (GARCH) MODEL}

Generally, the $\operatorname{ARMA}(a, b)$ process can be expressed in the following form: $\phi(B) p_{t}=\theta(B) \epsilon_{t}$; where $\left\{p_{t}\right\}_{t=1}^{T}$ is the time series, $\phi$ and $\theta$ are respectively $a^{\text {th }}$ and $b^{\text {th }}$ degree polynomials and B is backward shift operator defined by $B^{j} p_{t}=p_{t-j}$. In addition, $\epsilon_{t}$ is white noise sequence with Normal distribution: $\epsilon_{t} \sim N\left(0, \sigma^{2}\right)$. The $\phi$ and $\theta$ are expressed as follows:

$$
\left.\begin{array}{l}
\phi(B)=1-\phi_{1} B-\phi_{2} B^{2}-\ldots-\phi_{a} B^{a}, \\
\theta(B)=1+\theta_{1} B+\theta_{2} B^{2}+\ldots+\theta_{b} B^{b},
\end{array}\right\}
$$

To produce stationarity the underlying time series should be differenced as many times as needed (the inclusion of the factors of the form $(1-B),\left(1-B^{24}\right)$ and $\left(1-B^{168}\right)$, having a hope that the process under study will be reduced to ARMA process). In addition, the logarithmic transformation of the original data might be needed to get more homogenous mean and variance. Then, after having the stationary process the second step is to identify the resulting ARMA process (the order of the $\phi(B)$ and $\theta(B)$ polynomials). The existing tools are autocorrelation $(\mathrm{ACF})$ and partial autocorrelation (PACF) functions of the original data. Fig. 3 shows the ACF and PACF of the logarithmic transformed price data from March 10, 2012 to March 10, 2013. If the partial autocorrelation function of an autoregressive process of order $a$ cuts off, its autocorrelation function has a tail off after lag $a$. Similarly, the partial autocorrelation function of a moving average process of order $b$ has a tail off after lag $b$, while its autocorrelation function cuts off. A mixed process is suggested if both functions tail off. In successive trials the newly built model can be refined studying the ACF and PACF of the residuals.

However, the assumption of constant variance not always holds. The class of models, where the assumption of constant variance will fail to hold, refers as heteroskedastic. Hence, the Autoregressive Conditional Heteroskedastic (ARCH) models
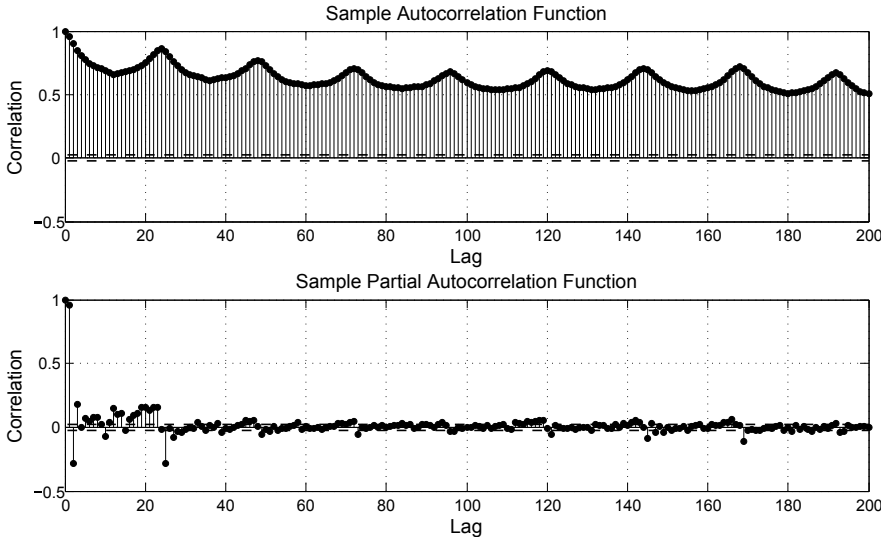

Fig. 3: ACF and PACF of input data

have been introduced to deal with the variance correlation throughout the time: $\operatorname{Var}\left(\epsilon_{t} \mid \epsilon_{t-1}\right)=V_{t}$. The $\operatorname{GARCH}(\mathrm{a}, \mathrm{b})$ is the extended version of $\mathrm{ARCH}(\mathrm{b})$ where;

$$
\epsilon_{t}^{2}=\nu_{t}^{2} V_{t}
$$

and $\sigma_{\nu}^{2}=1$ is white noise process

$$
V_{t}=c+\sum_{i=1}^{a} \psi_{i} V_{t-i}+\sum_{i=1}^{b} \Psi_{i} \epsilon_{t-i}^{2} .
$$

The GARCH model parameters for day-ahead market prices are estimated using GARCH package in MATLAB and are set out in Table I.

TABLE I: Estimated parameters for GARCH model; day-ahead ma
\begin{tabular}{l|l|l|l}
\hline Parameter & Estimate & Parameter & Estimate \\
\hline$\phi_{1}$ & 0.868 & $\phi_{48}$ & -0.02 \\
\hline$\phi_{2}$ & -0.25 & $\phi_{96}$ & 0.07 \\
\hline$\phi_{20}$ & -0.1 & $\phi_{168}$ & -0.06 \\
\hline$\phi_{21}$ & 0.15 & $\theta_{1}$ & 0.022 \\
\hline$\phi_{22}$ & -0.05 & $\theta_{2}$ & -0.004 \\
\hline$\phi_{23}$ & 0.12 & $\theta_{3}$ & 0.04 \\
\hline$\phi_{24}$ & 0.23 & $\theta_{4}$ & -0.01 \\
\hline$\phi_{25}$ & -0.28 & $\theta_{5}$ & -0.02 \\
\hline$\psi_{1}$ & 0.3 & $\psi_{2}$ & 0.04 \\
\hline$\Psi_{1}$ & 0.7 & & \\
\hline
\end{tabular}

The model validation is performed based on the diagnostic check, which tests whether the hypothesis about the residuals are true: residuals must be uncorrelated, normally distributed with 0 mean. For that purpose ACF and PACF plots of the residuals are studied Fig. 4. The ACF and PACF of the residuals together with the $\pm 1.96 / \sqrt{n}$ confidence bounds can be used to study how good the model can reflect the reality. [-1.96;1.96] is the confidence interval in which a measurement or trial falls corresponding to probability $=0.95$. This means that $95 \%$ of the autocorrelation values will lie inside the bounds and only $5 \%$ of autocorrelation values can be expected to fall outside of the bounds.

Besides, the Ljung-Box test is applied for residual autocorrelation. The test assesses the null hypothesis that a sequence of residuals does not show any autocorrelation for a fixed 


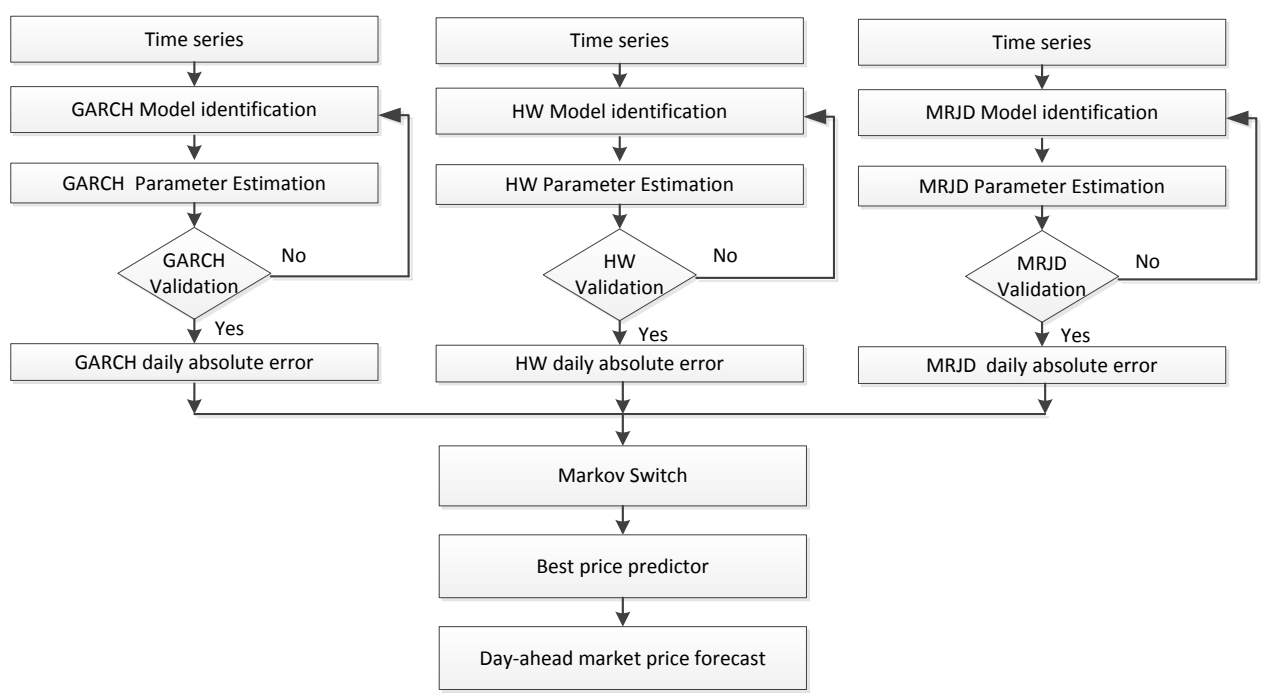

Fig. 2: Flowchart presenting steps in the whole framework.

number of lags $\mathrm{L}$, in contrast to the alternative hypothesis that there are some nonzero autocorrelation coefficients. After the validation step, the model can be used to predict and calculate daily absolute error.

One week (March 3 to March 10, 2013) real and simulated data for GARCH model is plotted in Fig. 5. The results show that the GARCH model is capable to follow day-ahead market prices. However, it is interesting to notice that, the GARCH model better fits to the last two days of the studied week in Fig. 5. For these two days the price variation is closer to the mean value.
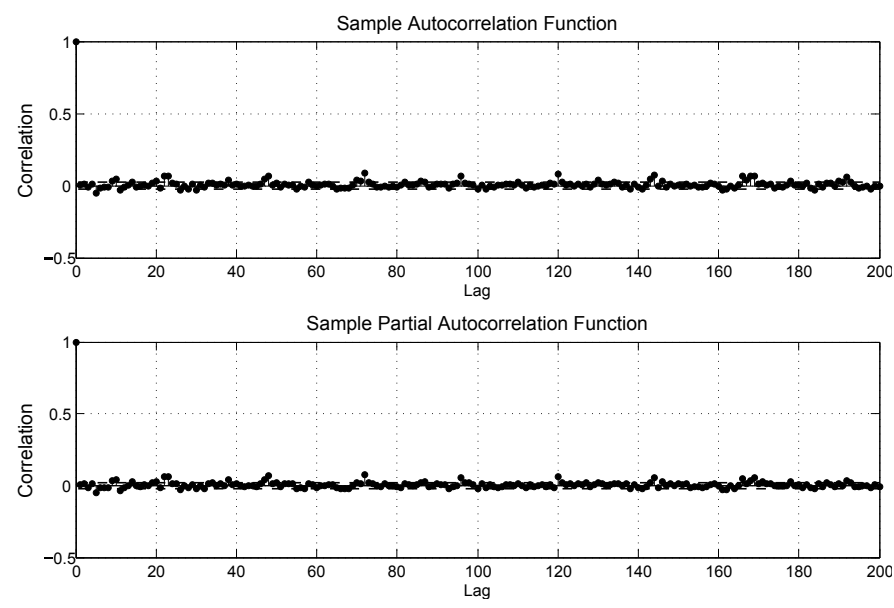

Fig. 4: ACF and PACF of reseduals

\section{MEAN REVERSION \& JUMP DIFFUSION (MRJD)MODEL}

The stochastic continuous-time MRJD process for a time series $\left\{p_{t}\right\}_{t=1}^{T}$ is defined as follows:

$$
d p_{t}=\left(\nu-\eta p_{t}\right) d t+\zeta d D_{t}+J_{t} d R_{t}
$$

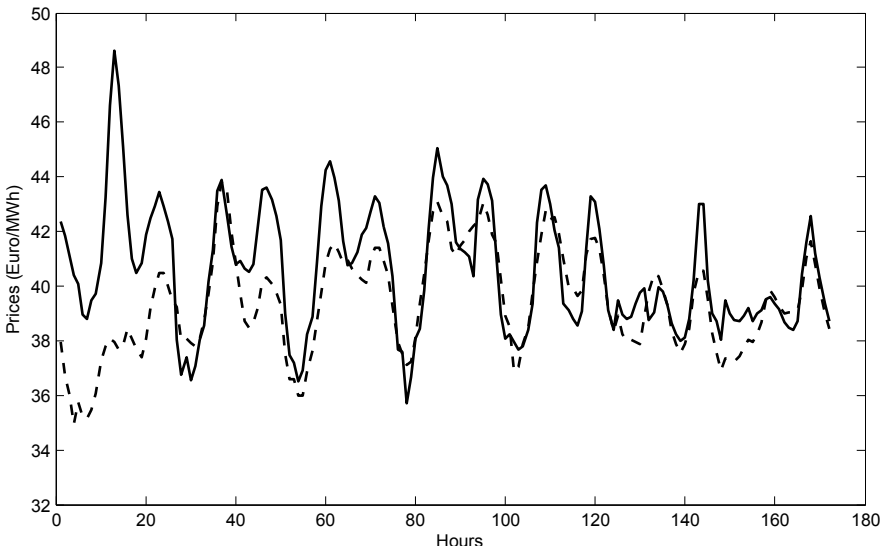

Fig. 5: One week real and simulated data for GARCH model: real data-solid curve and simulated data-dashed curve

In (4), $\frac{\nu}{\eta}$ is the long term mean. The $D_{t}$ is Brownian motion, which is responsible for frequent small fluctuations around $\frac{\nu}{\eta}$ proportional to $\zeta$. The Poisson process $R_{t}$ is producing nonfrequent big spikes of size $J_{t}$, which is normally distributed $N(\mu, \xi)$ with arrival rate of $\lambda[12]$.

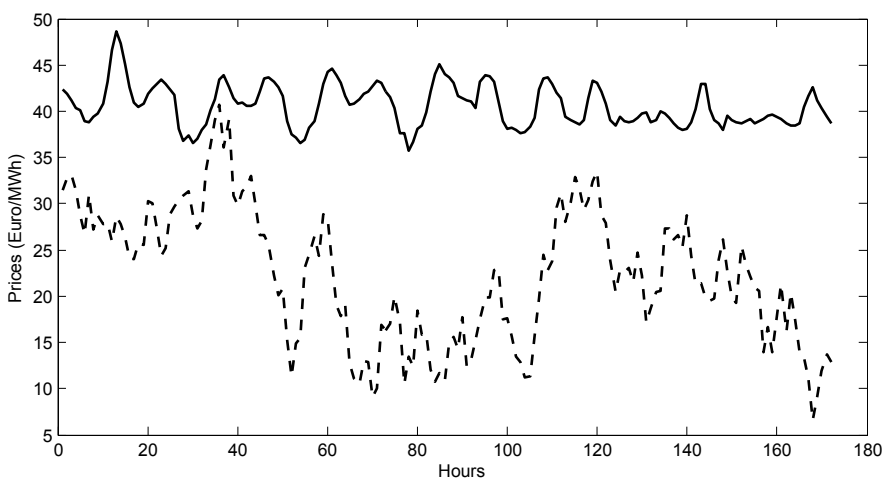

Fig. 6: One week real and simulated data for MRJD model: real data-solid curve and simulated data-dashed curve 
The parameters of MRJD model are derived using developed MATLAB codes in [12]. For the MRJD method, the corresponding parameters for day-ahead market are estimated as follows: long term mean parameters $\nu=1.04, \eta=0.04$, the parameter corresponding to frequent small fluctuations $\zeta=2.86$, the mean and the standard deviation of non-frequent spikes are $\mu=31.5$ and $\gamma=2.06$, and the corresponding arrival rate $\lambda=0.0034$.

Using estimated parameters daily absolute errors for day-ahead market prices can be calculated.

One week realized and evaluated energy prices are drawn in Fig. 6. Fig. 6 shows that MRJD model, provided in reference [12], fails to follow the real day-ahead market prices independent of the daily market price dynamics.

\section{Holt Winter (HW) MODEL}

The standard HW model for a time series $\left\{p_{t}\right\}_{t=1}^{T}$ with a unique seasonal pattern is as follows, [8], [13]:

$$
\begin{gathered}
\gamma_{t}=\alpha\left(p_{t} / I_{t-s}\right)+(1-\alpha)\left(\gamma_{t-1}+T_{t-1}\right) \\
T_{t}=\beta\left(\gamma_{t}-\gamma_{t-1}\right)+(1-\beta) T_{t-1} \\
I_{t}=\delta\left(p_{t} / \gamma_{t}\right)+(1-\delta) I_{t-s} \\
\tilde{p}_{t}(h)=\left(\gamma_{t}+h T_{t}\right) I_{t-s+h}
\end{gathered}
$$

Where $\gamma_{t}$ is the exponential component, $T_{t}$ is the trend and $I_{t}$ is the seasonal component with period $s$. The $\alpha, \beta$ and $\delta$ are smoothing parameters, which belong to the interval $[0,1]$. $\tilde{p}_{t}(h)$ is the forecast for $h$ hours forward.

The day-ahead market HW model is coded in MATLAB and estimated parameters are $\alpha, \beta$ and $\delta=0.1$.

In order to determine the forecasting power for HW method, realized and evaluated energy prices are depicted on Fig. 7 for the period March 3 to March 10, 2013. According to Fig. 7, HW model follows the price dynamics throughout the studied week. Fig. 7 shows that, in contrast to GARCH model, HW model best fits to the first five days in Fig. 7, where daily prices have two peaks. The HW model error is higher for those days where price variation is close to the mean (last two days in Fig. 7. From this comparison it is possible to notice, that one forecasting method fails to provide a good fit to all type of the days. To capture this regime switching behaviour, we propose a switch based on Markov model.

The second look on the Fig. 5 and Fig. 7 provides interesting insight. According to Fig. 5, GARCH model performs better for the last two days of the week, which are the weekend days. Obviously, these two days have different hourly price dynamics compared to the rest of the days in Fig. 5. Two question arise, (1) Are hourly price dynamics similar for all weekend days? (2) Does GARCH model perform better for all weekend days ?. To get the answers for those questions, two weeks realized and evaluated electricity prices (from February 24, 2013 to March 10, 2013) are drawn in Fig. 8 respectively. From both figures the real data curve shows that, hourly price dynamics for the week 1 weekend days (day 6 and day 7) are different from that of for the week 2 weekend days (day

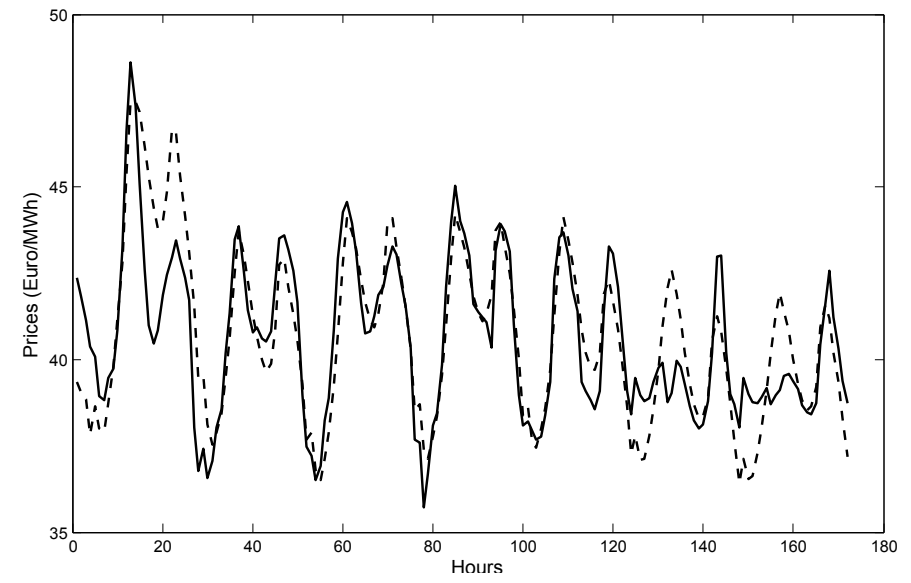

Fig. 7: One week real and simulated data for HW model: real data-solid curve and simulated data-dashed curve

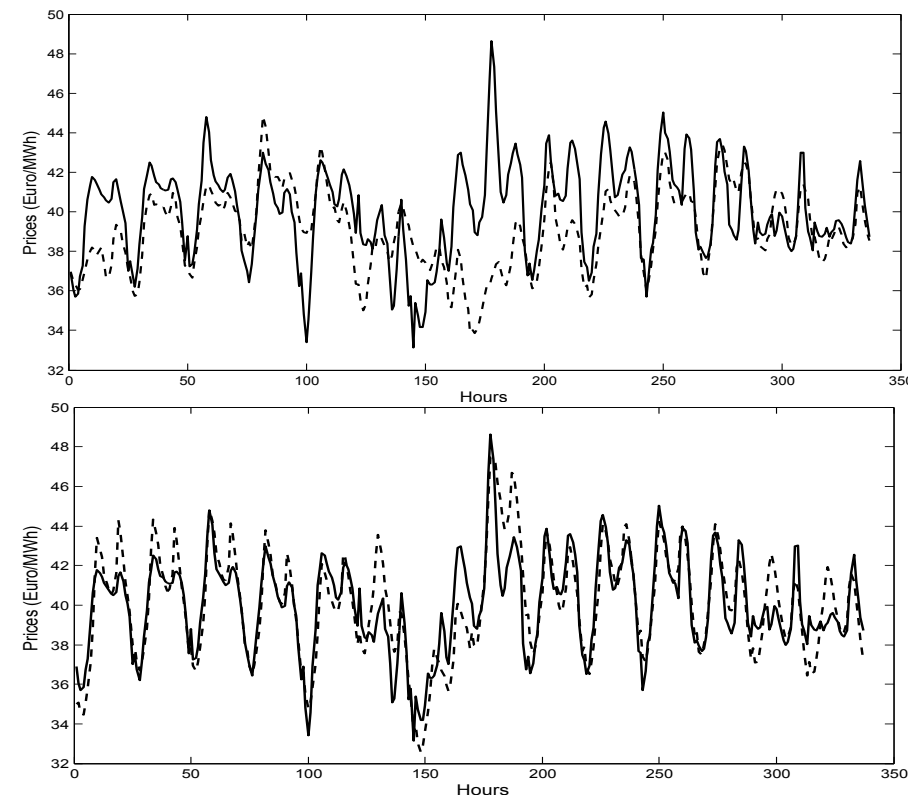

Fig. 8: Two weeks simulated data with GARCH (upper figure) and HW (lower figure) methods:: real data-solid curve and simulated data-dashed curve.

13 and day 14). The Fig. 8 illustrates that (upper figure), the GARCH forecasting model fits good for the week 2 weekend days, however it fails to follow the hourly price variation for the week 1 weekend days. For the week 1 weekend days the HW model performs better (see Fig. 8; lower figure). Thus, we cannot conclude that weekend days have similar hourly price dynamics and the GARCH model provides better fit for all weekend days. Moreover, the hourly price variation for some days is uncertain, hence which forecasting model will provide a better fit is quite unpredictable. Therefore, the advice for the best price predictor from Markov switch will increase day-ahead market price forecasting quality and accuracy.

\section{MARKOV SWITCH}

Suppose a set of $N$ competing price predictors producing forecasts $p_{t}^{(1)}$ to $p_{t}^{(N)}$ of real price $\hat{p}_{t}$. If $\left\{\epsilon_{t}^{(1)}, \ldots, \epsilon_{t}^{(N)}\right\}$ is the set of daily errors for price predictors 1 to $N$, then minimum 
element of this set $\left(\epsilon_{t}^{*}\right)$ determines the best performing price predictor for period t. The set of $\left\{\epsilon_{t}^{(1)}, \ldots, \epsilon_{t}^{(N)}\right\}$ can be used to design a Markov model which can predict the best performing predictor for period $t$ in the multi-predictor model. Specifically, let $\epsilon^{G A R C H}, \epsilon^{H W}$, and $\epsilon^{M R J D}$ be absolute errors for GARCH, HW and MRJD forecasting techniques as compared to the real prices. The parameter $e_{t}$ defines the state of Markov model at time $t$ :

$e_{t}=\left\{\begin{array}{llll}1, & \text { if } \epsilon^{G A R C H}<\epsilon^{H W} & \text { and } & \epsilon^{G A R C H}<\epsilon^{M R J D} \\ 2, & \text { if } \epsilon^{H W}<\epsilon^{G A R C H} & \text { and } \epsilon^{H W}<\epsilon^{M R J D} \\ 3, & \text { if } \epsilon^{M R J D}<\epsilon^{H W} & \text { and } \epsilon^{M R J D}<\epsilon^{G A R C H}\end{array}\right.$

Let $E_{i j}=\left\{e_{t}: e_{t}=j, e_{t-1}=i, t=1, \ldots, T\right\}$, then the $3 \times 3$ transition probabilities matrix is defined as:

$$
\operatorname{pr}_{i j}^{S W}=\frac{\operatorname{Card}\left(E_{i j}\right)}{\sum_{n=1}^{3} \operatorname{Card}\left(E_{i, n}\right)}, i, j=1, \ldots 3
$$

Using estimated parameters the daily absolute error is calculated comparing the real prices and the simulated prices applying all three forecasting techniques for the whole year. Then, diurnal absolute errors using all three forecasting tools are depicted in Fig. 9. Entering daily absolute error series for all forecasting models as input data to Markov switch model we can get transition probability matrix (11).

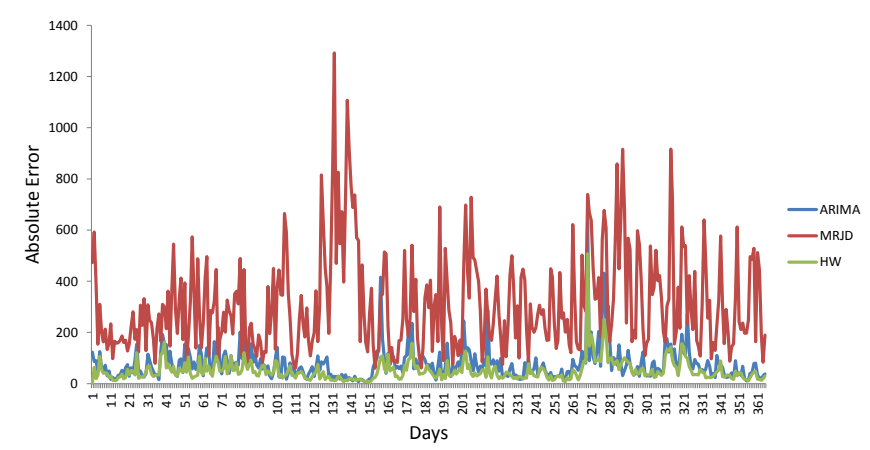

Fig. 9: The daily absolute error for ARIMA, MRJD and HW methods

$$
P_{\text {switch }}=\left(\begin{array}{ccc}
0.2619 & * & 0.7381 \\
* & * & * \\
0.2222 & * & 0.7786
\end{array}\right)
$$

According to Fig. 9 , $23 \%$ of cases ARIMA method outperforms and $77 \%$ cases HW method beats its competitors. This behaviour is also consistent with (11). In addition, both Fig. 9 and transition probability matrix in exposed in (11) clearly shows that, the MRJD happened to be the worst predictor for our price data. We have drawn this conclusion also by analyzing Fig. 6.

Using the transition matrix above, we can determine the best predictor for forecasting the next day prices. According to the results, Markov switch selects HW model as the best fit for the hourly price dynamics happening in March 11, 2013. Real and predicted curves using all forecasting tools for March 11, 2013 are depicted in Fig. 10. Fig. 10 shows that HW forecasting model outperforms its competitors.

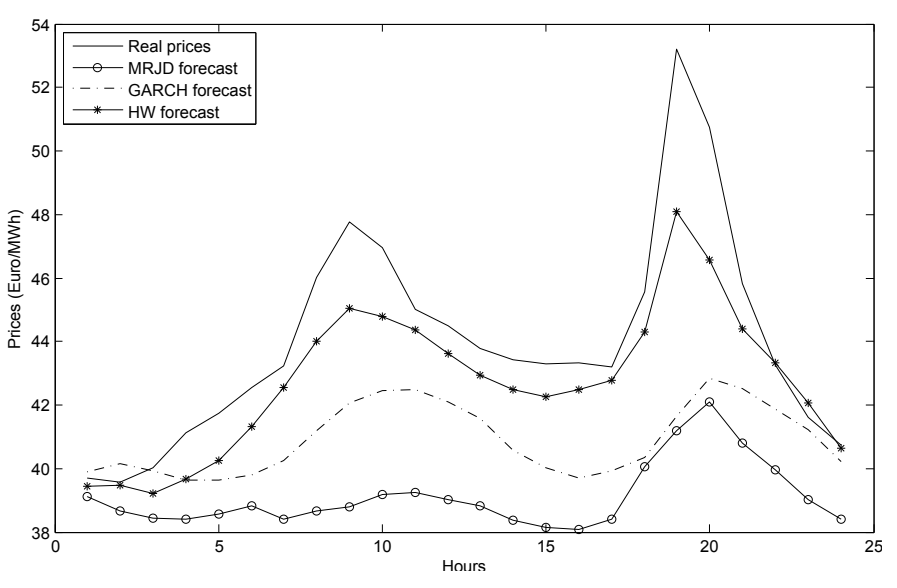

Fig. 10: Real and forecasted curves

In addition, the MAPE (Mean Absolute Percent Error) values for three forecasting methods are provided in Table II. The MAPE measures the size of the error in percentage term. As Table II shows the MAPE value is smaller for the HW forecasting technique. The results from Table II are consistent with the results depicted in Figure 10.

\section{CONCLUSION}

This paper presents three day-ahead market price forecasting methods (GARCH, HW and MRJD) based on time series analysis, exponential-smoothing and stochastic processes methodologies. The Markov switch model predicts the bestprice model for forecasting the day-ahead market prices in the day of planning. This means one of the alternative forecasting models is chosen as the best one for the planning day. All three forecasting model performances are tested using one year historical data from Nordic Electricity market. The Markov switch is run to detect the forecasting tool which outperforms its competitors. According to the results, $77 \%$ of the time HW is detected as the best forecasting tool, while the GARCH model performs better for only $23 \%$ of the cases. The MRJD method is recognized as the worst forecasting technique to predict day-ahead market prices within three mentioned alternatives.

\section{REFERENCES}

[1] S. Fiorenzani, Quantitative methods for electricity trading and risk management. Palgrave macmillan, 2006.

[2] Nord Pool database (http://www.nordpoolspot.com).

[3] K. S. Aggarwal, M. Saini, and A. Kumar, "Electricity price forecasting in deregulated markets: A review and evaluation," Electrical Power and Energy Systems, vol. 31, no. 1, pp. 13-22, January 2009.

[4] G. E. P. Box and G. M. Jenkins, Time Series Analysis Forecasting and Control. Holden-Day, 1976.

[5] P. J. Brockwell and R. A. Davis, Time Series: Theory and Methods. Springer-Verlag Inc. New York, 1991.

[6] J. Contreras, R. Espinola, F. J. Nogales, and A. Conejo, "ARIMA models to predict next-day electricity prices," IEEE Transactions on Power Systems, vol. 18, no. 3, pp. 1014-1020, July 2003. 
TABLE II: The MAPE values for three forecasting methods

\begin{tabular}{|c|c|c|c|c|c|c|c|}
\hline Hour & Actual & MRJD & GARCH & HW & Absolute \% error & Absolute \% error & Absolute \% error \\
\hline & data & forecast & forecast & forecast & MRJD & GARCH & $\mathrm{HW}$ \\
\hline 1 & 39.69 & 39.12 & 39.88 & 39.43 & 1.44 & 0.48 & 0.66 \\
\hline 2 & 39.58 & 38.66 & 40.16 & 39.46 & 2.33 & 1.46 & 0.31 \\
\hline 3 & 40.03 & 38.44 & 39.92 & 39.21 & 1.59 & 0.27 & 2.13 \\
\hline 4 & 41.11 & 38.41 & 39.63 & 39.68 & 6.57 & 3.59 & 3.72 \\
\hline 5 & 41.75 & 38.57 & 39.64 & 40.26 & 7.61 & 5.05 & 3.86 \\
\hline 6 & 42.55 & 38.84 & 39.80 & 41.31 & 8.72 & 6.47 & 3.19 \\
\hline 7 & 43.24 & 38.40 & 40.26 & 42.54 & 11.20 & 6.90 & 1.82 \\
\hline 8 & 46.01 & 38.65 & 41.18 & 44.02 & 16.00 & 10.50 & 5.15 \\
\hline 9 & 47.77 & 38.78 & 42.06 & 45.05 & 18.81 & 11.95 & 7.01 \\
\hline 10 & 46.94 & 39.18 & 42.47 & 44.77 & 16.54 & 9.53 & 5.54 \\
\hline 11 & 45.02 & 39.23 & 42.50 & 44.35 & 12.85 & 5.61 & 1.71 \\
\hline 12 & 44.48 & 39.02 & 42.09 & 43.63 & 12.27 & 5.38 & 2.18 \\
\hline 13 & 43.78 & 38.81 & 41.58 & 42.94 & 11.35 & 5.03 & 2.16 \\
\hline 14 & 43.41 & 38.37 & 40.57 & 42.49 & 11.61 & 6.55 & 2.40 \\
\hline 15 & 43.29 & 38.16 & 40.03 & 42.26 & 11.86 & 7.52 & 2.70 \\
\hline 16 & 43.33 & 38.09 & 39.69 & 42.48 & 12.09 & 8.40 & 2.23 \\
\hline 17 & 43.2 & 38.41 & 39.93 & 42.78 & 11.09 & 7.57 & 1.09 \\
\hline 18 & 45.56 & 40.04 & 40.36 & 44.29 & 12.11 & 11.41 & 3.17 \\
\hline 19 & 53.22 & 41.18 & 41.65 & 48.09 & 22.63 & 21.74 & 12.46 \\
\hline 20 & 50.74 & 42.09 & 42.83 & 46.58 & 17.04 & 15.59 & 9.88 \\
\hline 21 & 45.83 & 40.80 & 42.52 & 44.39 & 10.97 & 7.22 & 3.53 \\
\hline 22 & 43.25 & 39.95 & 41.89 & 43.33 & 7.64 & 3.16 & 0.20 \\
\hline 23 & 41.61 & 39.02 & 41.21 & 42.08 & 6.22 & 0.95 & 1.20 \\
\hline 24 & 40.73 & 38.41 & 40.23 & 40.63 & 5.70 & 1.24 & 0.26 \\
\hline МАPE & & & & & 10.78 & 6.81 & 3.27 \\
\hline
\end{tabular}

[7] R. C. Garcia, J. Contreras, M. van Akkeren, and J. B. C. Garcia, "A GARCH forecasting model to predict day-ahead electricity prices," IEEE Transactions on Power Systems, vol. 20, no. 2, pp. 867-874, May 2005.

[8] P. Winters, "Forecasting sales by exponentially weighted moving averages," Management Science, vol. 6, no. 3, pp. 324-342, April 1960.

[9] V. Lepojevic and M. Andelkovic-Pesic, "Forecasting electricity consumption by using holt-winters and seasonal regression models," Economics and Organization, vol. 8, no. 4, pp. 421-431, 2011.

[10] T. Jonsson, P. Pinson, A. H. Nielsen, and H. Medsen, "Exponential smoothing approaches for prediction in real-time markets," Energies, vol. 7, no. 6, pp. 3710-3732, June 2014

[11] R. Weron, M. Bierbrauer, and S. Truck, "Modeling electricity pricees: jump diffusion and regime switching," Hugo Steinhous Center for Stochastic Methods Wroclaw University of Technology, Tech. Rep., 2001.

[12] R. Weron, "Matlab function to estimate, simulate and predict of a meanreverting jump-diffusion (mrjd) process," in Boston College Department of Economics, 2010.

[13] J. W. Taylor, "Short-term electricity demand forecasting using double seasonal exponential smoothing." Journal of the Operations Resaerch Society, vol. 54, no. 8, pp. 799-805, August 2003. 